\title{
Exploring and Mapping Indoor Environment for Mobile Robots using Different Ways of Scanning
}

\author{
Prof. Sohair F. Rezeka \\ Mechanical Engineering Department \\ Arab Academy for Science, Technology and Maritime \\ Transport \\ Alexandria, Egypt
}

\author{
Mohamed Mahrous Hassanien \\ Mechanical Engineering Department \\ Arab Academy for Science, Technology and Maritime \\ Transport \\ Alexandria, Egypt
}

\begin{abstract}
Exploring and mapping for any unknown indoor environment is one of the main topics that are currently been exploring all over the globe. However, A fully developed automated system for exploring and mapping indoors is not yet implemented commercially, although there are currently some systems that works automatic but partially or it misses some data from the surroundings which gives false statements. Also, in some scenarios some systems take plenty of time because of the huge processing data that is resulted from the sensors used. In this paper, we will demonstrate a complete process from exploring the indoor environment (Forward tracking) and mapping or path planning for the point that we desire to reach (Back tracking). A developed Kinematic model of a Robotino Wheeled Mobile Robot (WMR) is going to be used to implement our work including scanning techniques as well as path planning techniques. furthermore, two scanning methods are going to be used, one by other, to get the best result in time and accuracy for them. The two sensors are a matrix of Infrared sensors (IR) that are fixed around the WMR body and a Light detection and ranging sensor (LiDAR). A comparison is going to be discussed for the best results of these sensors.
\end{abstract}

Keywords-WMR , IR, LiDAR , path planning , Forward tracking, Back tracking

\section{INTRODUCTION}

Fully automated mobile robots are becoming a major research point in the field of robotics industry world widely as stated in 2018 report of the international federation of robotics [1]. As it was ensured that some countries are at the forefront of experimenting and developing of the technologies and methods that will ultimately lead to the goal of fully automated robots. The technology will be a real game changer of the roads, delivering a real impact on the speed and accuracy of the work. Eliminating the Human errors - which reported to be responsible for more than $80 \%$ of production failures- is one of the main factors why most of the industry is turning towards this technology [2]. As appeared in Figure 1, Huge amount of spending on researches and publications were published studying all the necessary scenarios to insure the safety and accountability of this technology.

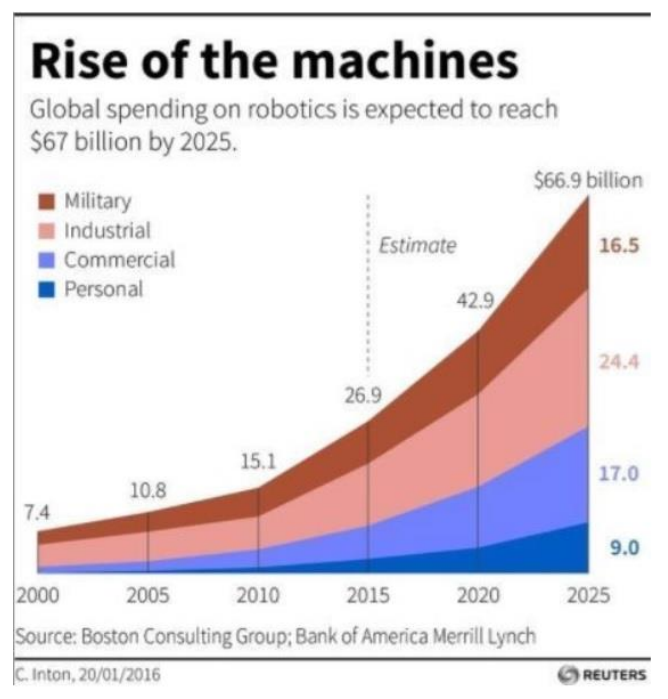

Figure 1 : increasing number of researches on robotics field.

Fortunately, the continuous advances in computer hardware and software helped for the continues development in this field and all the tracks are being tackled from different points of view to reach the desired results. One main issue that is facing the current technology are to discover the environment around the robot and to find a safe path between two known points after knowing how the area around the robot looks like, the system then should lead the robot through any routes or environmental condition. Searching the environment is called mapping or forward tracking, while helping the robot to reach its destination is called path planning or back tracking. Mapping is known as the ability to autonomously build a plan by using data gathered from various systems or sensors. While Path planning is particularly defined as reaching the defined target from a known point.

In this paper, A fully developed system will be presented starting from mapping for indoor environment along with path planning feature. A Robotino WMR is going to be used to validate our work. Robotino has its own simulation software which is going to be connected to LABView and Matlab in order to use a kinematic model that is developed specially for this project. A Comparison in time of exploring will be conducted between two scanning sensors, one with a LiDAR sensor and the other using 9- Infrared sensors installed around the circumference of the Robotino as shown in Figure 2. 


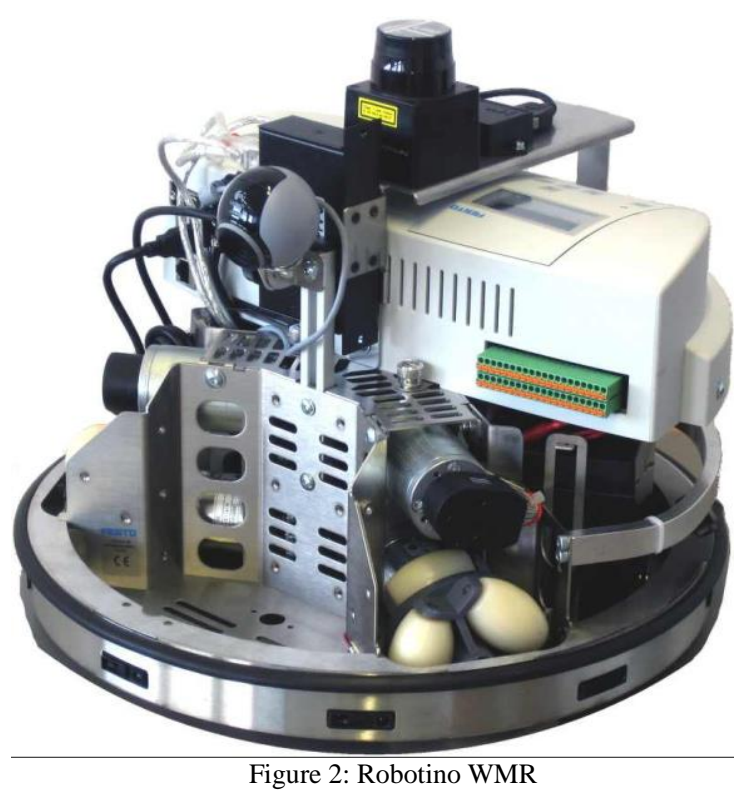

Mapping is the ability for an autonomous robot to construct a map or plan by collecting the environment data using various sensors[3]. Many approaches and algorithms have been proposed to serve the process of mapping such as: Open Path algorithm[4][5], Behaviors-based exploration (BBC) algorithm[6][7], Rapidly-exploring random tree (RRT) algorithm[8] and Sensor-based random tree (SRT) algorithm [9][10].

After getting a good vision of the area (Forward tracking) a path planning is required to reach our targeting point. A lot of methods are developed for this purpose as A-star search algorithm [11][12] and Dijkstra's algorithm[13].

A kinematic model was developed for Robotino WMR to validate the work. The model is based on paper [14] and developed by Bayoumi [15]. More details to be discussed in following sections.

The following sections will be divided as follows: Section II, an overall approach, hardware and software configurations will be discussed. Section III will contain the detailed explanation of the process including data collection from LIDAR, data processing and path planning. In Section IV, Simulation results and experimental work results will be discussed. Finally, the conclusions and future work will be discussed in Section V.

\section{APPROACH}

The purpose of this paper is to investigate the process of using different types of sensors for doing a mapping for indoor environment. A fully automated system is developed, modelled and simulated. There will be four major areas of study in this process, Data collection, Forward tracking, Backward tracking and the main control system as shown in Figure 3. In data collection part, the data will be gathered from different sensors (9-IRs OR LiDAR, Encoders, Odometry, etc..) to be processed in order to be ready for using in the forward and backward tracking techniques.

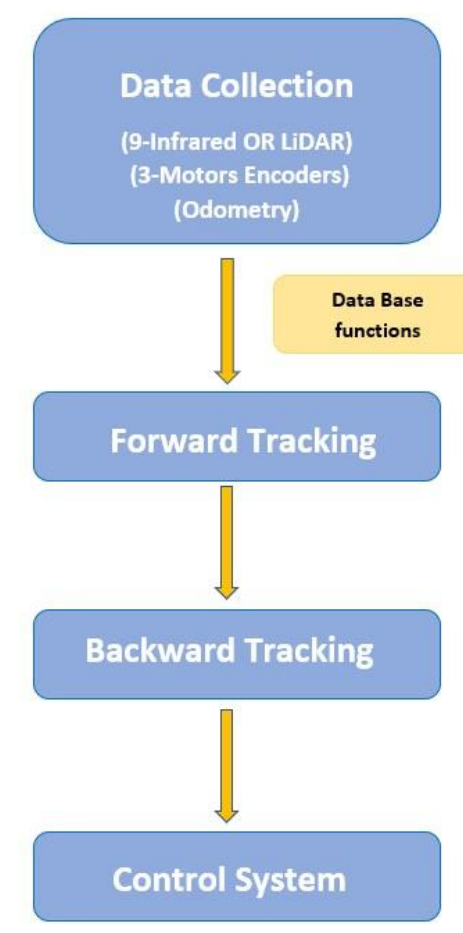

Figure 3: main approach for the research paper

In data processing, the collected data will be managed and simplified so that we can get the distance and angle for the Obstacle location in the fore-path of Robotino, these data will be analyzed and used in the tracking algorithms.

In Figure 4, the LiDAR data is processed and used to analyze the location of the walls, obstacles and corners. These data will be used to draw a map for the environment. Afterwards, the targeted location will be set, and it will be reached through these obstacles which originally found while discovering the place. The main method that we are going to use in this paper is Dijkstra search algorithm.

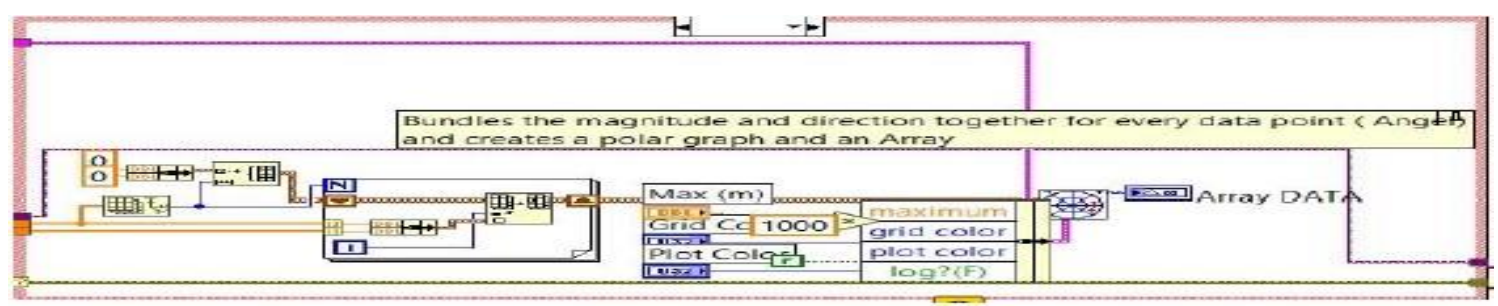

Figure 4: LiDAR data processing 
The last step is to prepare and send the data of the path which is generated from the path planning algorithm with consideration of the road/area mapping constrains and obstacles. Our model is going to be used along with Robotino official Simulator to validate the work on.

For the hardware configuration, Robotino View2 robot and its simulation will be used, 9 infrareds installed around the chase, RPLiDAR as the laser range finder and built in Odometry located on the robot itself. Data Processing, Mapping and path planning are going to be implemented as well as the control system using LABVIEW, MATLAB and Robotino View2 Simulator. The three softwares will be connected to each other's to present a real time simulation. Also, the Robotino View2 has its own controller which connects wireless through WIFI to the PC.

\section{IMPLEMENTATION}

This section concerns with implementing the required process to achieve our project's target. The project control system is divided into minor levels for the ease of design. In Figure 6, The general control system of exploration task converts complex system to multi simple levels or functions in hierarchical control levels starting from user level to the Control level. This system is programmed by "LABVIEW Software" and simulated by "Robotino SIM professional".

Starting by the control level, which can be assigned as the axis-level. This level contains the microprocessor which controls the Robotino by (PC 104 microprocessor). It's a built-in processor inside the control system. In this level, the data are collected from the 9-IRs and the LiDAR to be sent to the upper level using wireless communication. Also, the robot has 3 motor encoders, which are connected to the controller. The controller converts motor rotations into pulses, which the last one is translated by the odometry into a position and send it to a higher level as well. The wheels of the robots are controlled from this level, the WMR controller adjusts the speeds of the three motors using PID algorithm as showing in Figure 5.The process value (feedback) is received from the encoder. The controller parameters, $\mathrm{Kp}, \mathrm{Ki}, \mathrm{Kd}$, are parameters calculated by Ziegler-Nichols method at supervisory control Error! Reference source not found.[16] and sent to WMR controller.

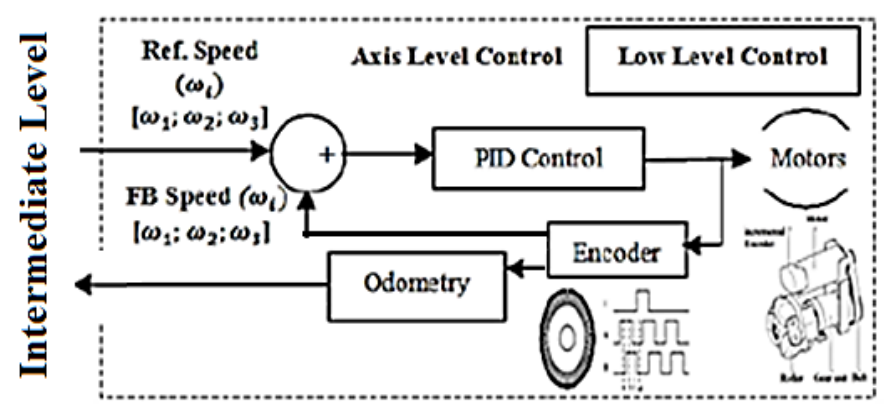

Figure 5 : Actuators control system

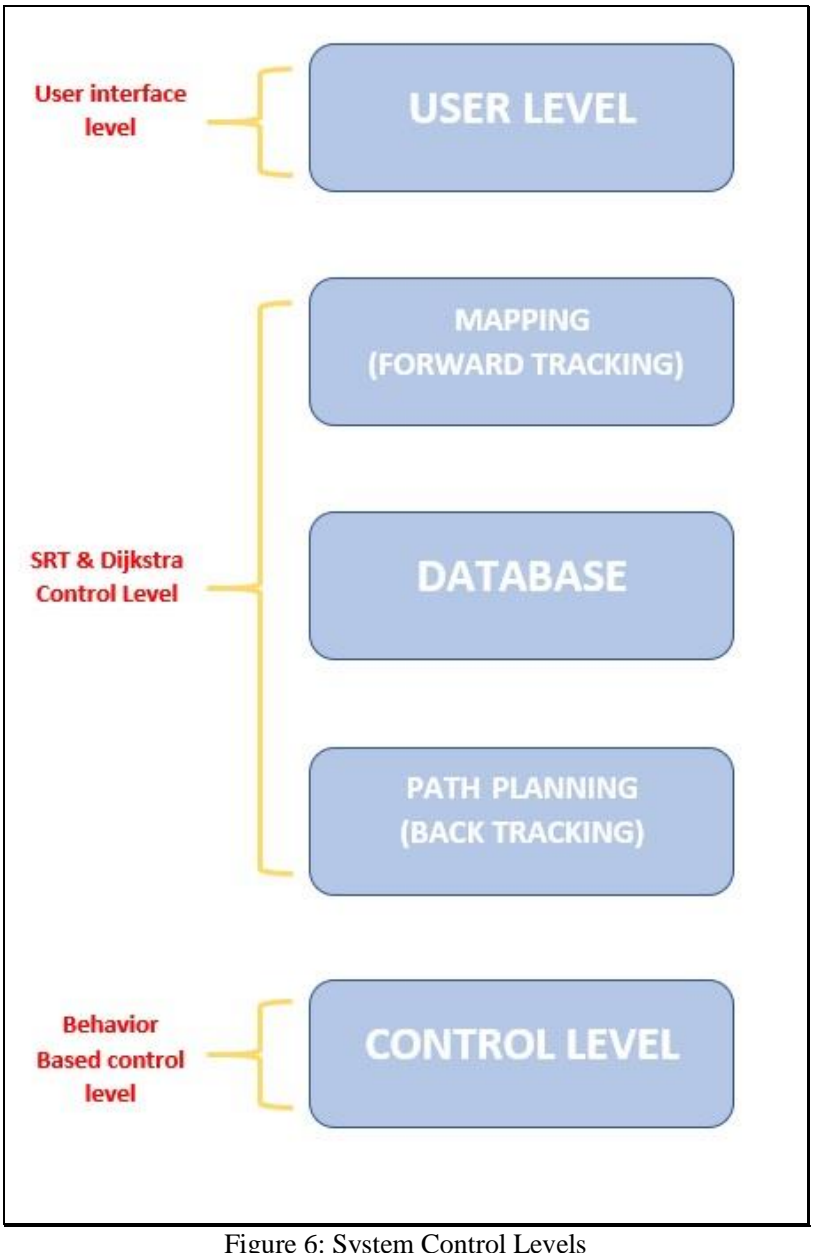

For the Intermediate level, which including simplifying the data coming from the control level to be suitable to use with the mapping (Sensor- Based Random Tree "SRT") and path planning (Dijkstra) Algorithms. This level is considered as a gate that allows the robot controller to be interfaced with LABVIEW software. A "Com function" function is developed for this purpose. In this level, there are three important main tasks which are: mapping, database and path planning.

For mapping, the robot will move to explore the surroundings by using dedicated sensors (9-IRs OR LiDAR). For the database level, it has two main functions: The "Storage function" which stores important data received from exploration and intermediate levels and the "Edit or Delete Data function" which is used to update the unexplored-angles table resulted at database during exploration.

For the path planning level, the WMR has a map and search for the shortest path to reach a target position. The "Dijkstra algorithm" is employed to determine the shortest path to the nearest explored target position that has unexplored angles.

User level is the top level in the supervisory control and it depends on required set from the user and how the user wants to manage the data and the robot. This level can be divided into two sections; user-control level and user- 
monitoring level. For the user control level, the user enters commands to the WMR controller by using control system software. This data may be numeric values, switches and selectors. Hence, the software must be easy to deal with and has good user interface. For the User monitoring level, the user can monitor the feedback data received from the robot controller and software.

\section{SIMULATION RESULTS AND CONCLUSION}

For this section, results will be represented for different rooms shaping, showing the difference between using the 9Infrared sensors and LiDAR sensor for the same configurations.

\section{A. Free room}

A simulated free room $3 \times 3 \mathrm{~m}$ were simulated and the robot was starting from the middle of the room Figure 7 and

Figure 8 clarify:

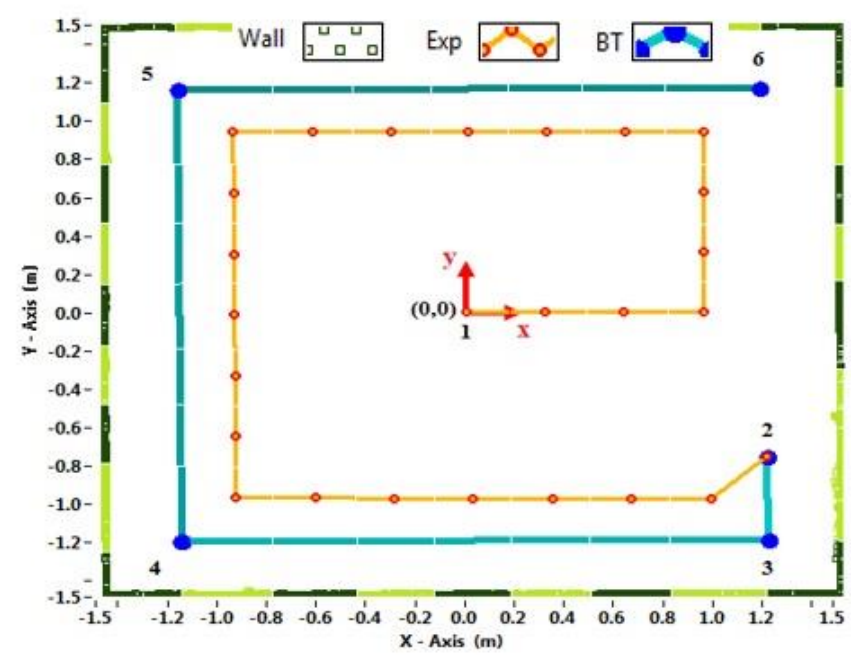

Figure 7: Free room mapping and backtracking using infrared sensors

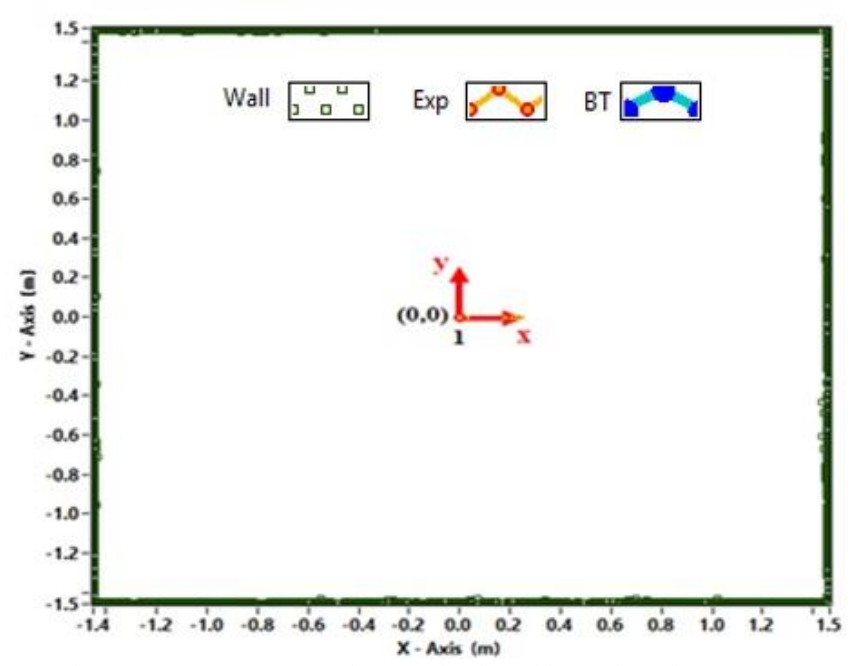

Figure 8: Free room mapping and backtracking using LiDAR sensor

The difference in time is quite big as the WMR didn't take more than 8 seconds compared for using the IRs 310.55 seconds. Of course, the high range of the LiDAR $(8 \mathrm{~m})$ is playing a good rule in this case compared with the IRs sensors $(4-40 \mathrm{~cm})$.

\section{B. Partitions and internal enterance room}

The outside dimensions of this place are $3 \times 3 \mathrm{~m}$ with inside partitions and internal entrance as shown in Figure 9 and Figure 10

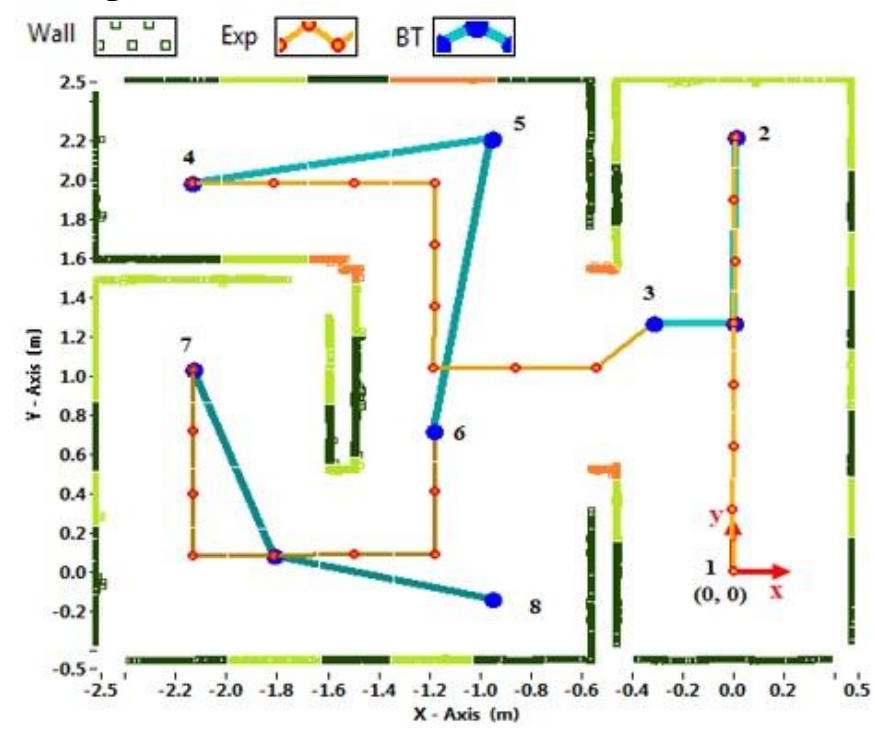

Figure 9: partitioned room mapping and back tracking using infrared sensors

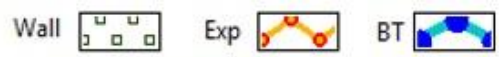

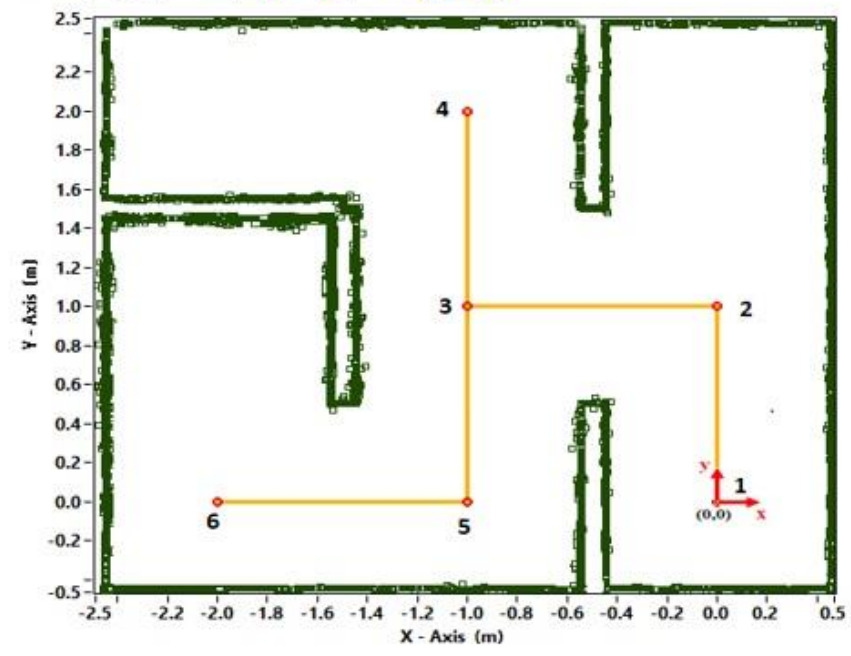

Figure 10: Partitioned room mapping and back tracking using LiDAR sensor

For partitioned room, the LiDAR was taking the lead in time (93 secs) compared to infrared sensors (301 secs).

\section{Zigzag room}

In this case, a zigzag room is simulated to test both sensors as shown in 


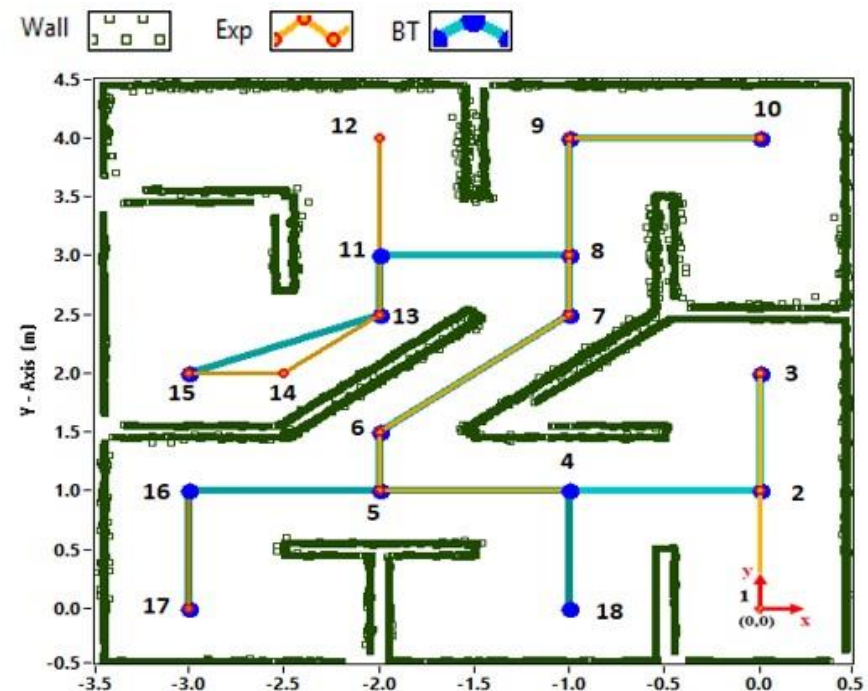

Figure 11: Zigzag room mapping and backtracking using LiDAR sensor

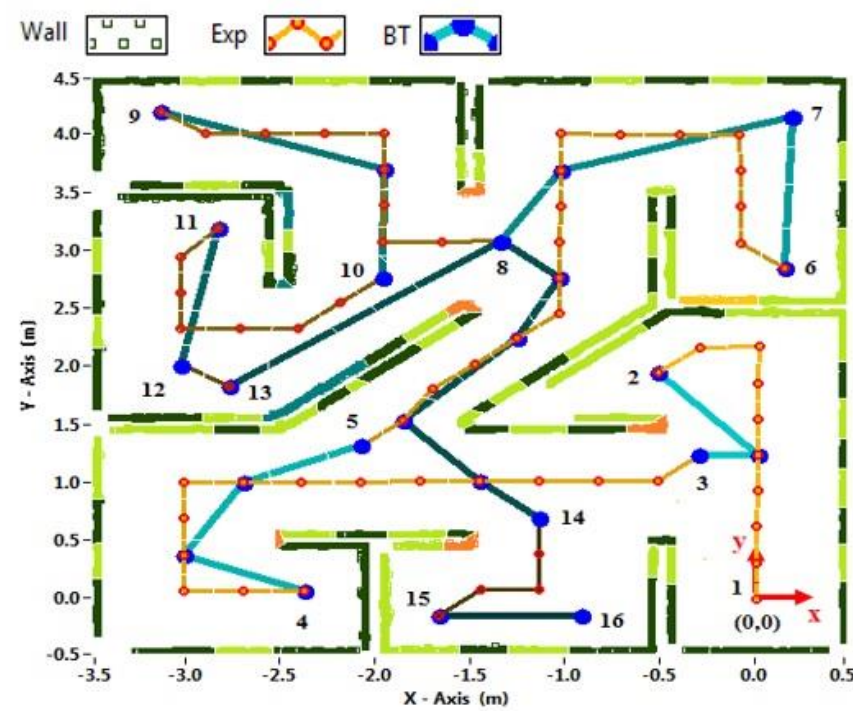

Figure 12: Zigzag room mapping and backtracking using IR sensors

For the zigzag room, the results were (432 secs) for LiDAR and (725 secs) for the IR sensors.

It is obvious that in the simulation results, the LiDAR have the advantage in time of scanning than the IR sensors, but this result will help in choosing between these two sensors in the required application depending on the time required to finish the task comparing to the project budget because there is a big difference in price between choosing between IR sensors and LiDAR sensor.

\section{FUTURE WORK}

A practical result will support the work and it is currently on going. Also, It would be good idea to implement this work on narrow areas and small obstacles which might not be detected by the LiDAR.

\section{REFERENCES}

[1] I. International Federation of Robotics, "Robots and the Workplace of the Future," no. March, p. 35, 2018.

[2] B. S. Dhillon, "Human error in maintenance: An investigative study for the factories of the future," IOP Conf. Ser. Mater. Sci. Eng., vol. 65, no. 1, 2014.

[3] B. Tovar, R. Murrieta-Cid, and C. Esteves, "Robot motion planning for map building," IEEE Int. Conf. Intell. Robot. Syst. vol. 1, pp. 673-680, 2002

R. Von Wahlde and M. A. J. N. Wiedenman, "An Open-Path Obstacle Avoidance Algorithm Using Scanning Laser Range Data," Army Res. Lab., no. February, 2009.

[5] N. Tuck, M. McGuinness, and F. Martin, "WOAH: an obstacle avoidance technique for high speed path following," pp. 787811$787811,2011$.

[6] B. Rodney, "Achieving artificial intelligence through building robots," no. October 2004, p. 11 p, 1986.

[7] R. A. Brooks, "A Robust Layered Control System For A Mobile Robot," IEEE J. Robot. Autom., vol. 2, no. 1, pp. 14-23, 1986.

[8] Z. Shiller, Motion and Operation Planning of Robotic Systems, vol. 29. 2015.

[9] "S. M. LaValle, 'Rapidly-exploring random trees: A new tool for path planning,' Computer Science Dept., Iowa State University,pp 98-11, October 1998."

[10] "S.M. LaValleand J. J. Kuffner, 'Rapidly-exploring random trees: Progress and prospects', Proc. of the Workshop on the Algorithmic Foundations on Robotics, 2000."

[11] "H. Antikainen, 'Using the Hierarchical Pathfinding A* Algorithm in GIS to Find Paths through Rasters with Nonuniform Traversa Cost,' ISPRS Int. J. Geo-Information, vol. 2, no. 4, pp. 996-1014, 2013."

[12] "D. Li, M. Liu, J. Zhang, and E. Cheng, 'An Improved A* Algorithm Applicable for Campus Navigation System,' Proc. 2015 Int. Conf. Netw. Inf. Syst. Comput. ICNISC 2015, pp. 588 591, 2015."

[13] S. Sivakumar and C. Chandrasekar, "Modified Dijkstra's Shortest Path Algorithm," Int. J. Innov. Res. Comput. Commun. Eng., vol. 2, no. 11, pp. 6450-6456, 2014.

[14] J. Wu, R. L. Williams, and J. Lew, "Velocity and," J. Dyn. Syst. Meas. Control, vol. 128, no. 4, p. 788, 2006

[15] M. Bayoumi, "Enhancement of Exploration and Backtracking Techniques of Wheeled Mobile Robot," Arab Acad. Sci. Technol. Thesis, p. 2017, 2017.

[16] K. Ogata, Modern Control Engineering, $5^{\text {th }}$ Edition. 